Vorstudie des IÖW

im Auftrag des DGB-Berlin und der Hans-Böckler-Stiftung

\section{Ökonomisch-ökologische Perspektiven für Berlin 2010}

Der DGB-Berlin und die Hans-Böckler-Stiftung planen gemeinsam mit dem IÖW ein umfangreiches Forschungsvorhaben, das die industriepolitischen Perspektiven Berlins für die kommenden 20 Jahre untersuchen soll. Eine Vorstudie zu diesem Forschungsprojekt von großer politischer Relevanz wurde von Heidi Fichter, Martin Jänicke und Rainer Knigge (unter Mitarbeit von: Harald Mönch; Koordination: Gabriel Knieschon) erstellt. Wir dokumentieren im folgenden die diese Vorstudie einleitende Kurzfassung:
Die vorliegende Untersuchung ist die Vorstudie zu einem langfristigen ökonomisch-ökologischen Entwicklungskonzept für Berlin (West). In der weiteren Forschung sollen im Sinne der Szenario-Methode zwei Entwicklungspfade dargestellt werden. Szenarien sind bedingte Prognosen. Sie sollen danach unterschieden werden, ob vorhandene Entwicklungspotentiale in einer ehrgeizigen ökologischen Strukturpolitik ausgeschöpft werden (Alternativ-Szenario) oder nicht (Trend-Szenario). Die Besonderheit der Hauptzierten Einflußfaktoren nicht nur Ökonomie und Ökologie, sondern auch die nicht-ökonomischen Rahmenbedingungen (z.B. als Ergebnis einer Politikprognose) einschließen.

Die hierzu erfolgte erste Prüfung von Langzeittrends und Strukturfaktoren in industriellen Ballungsräumen ergibt für Berlin Rückständigkeiten, die durch ein ökonomisch-ökologisches Strukturkonzept erst einmal beseitigt und schließlich überkompensiert werden müssen.

Dies ist einer der Angelpunkte der Untersuchung: Die Stadt hat ihren Standortnachteil in keiner Weise überwunden und ist in das Umland ökonomisch (mit einem Anteil des DDR-Exports von 1,4 Prozent) nicht integriert. Sie besitzt als Folge der Berlinförderung eine äußerst ungünstige Wirtschaftsstruktur. Und auch der Energieverbrauch, die Verkehrsstruktur und die Flächenstruktur erweisen sich als atypisch für eine gehend als Folge dieser Anomalien - eine vergleichsweise ungünstige Beschäftigungssituation und erhebliche ökologische Standortnachteile. Im Trend-Szenario wird unterstellt, daß - ungeachtet vielfacher Bemühungen - der skizzierte strukturelle Rückstand nicht überwunden wird. Das Alternativ-Szenario untersucht hingegen Bedingungen, die einen Entwicklungs- und Instudie soll u.a. darin liegen, daß die prognostimoderne Industrieregion. Hinzu kommen - weit- novationsdurchbruch hin zu einer Vorreiterrolle der Stadt in ökologischer und technologischer Hinsicht ermöglichen.

Für das Alternativ-Szenario wird das Konzept einer ökologischen Strukturpolitik entwickelt. Gemeint ist ein gleichermaßen ökologisches wie ökonomisches Langzeitkonzept. Umweltpolitisch zielt es auf eine Überwindung der Defizite des entsorgenden Umweltschutzes in Form von nachgeschalteten Umweltentlastungstechnologien und-maßnahmen. Ökonomisch zielt es auf die Überwindung einer kostenträchtigen Produktionsweise, die knappe Ressourcen extensiv in Schadstoffe und Abfälle umwandelt. Im Kern handelt es sich beim Alternativ-Szenario um das objektiv mögliche Resultat einer Innovationsstrategie, deren Vorteile auch im Bereich der Arbeitsmarktpolitik liegen: Innovative Produktionen sind in aller Regel arbeitsintensiver als standardisierte Massenproduktionen, wie sie die Berlinförderung begünstigt. Die technische Einsparung stofflicher Inputfaktoren der Produktionen ist z.T. eine Alternative zur Einsparung des Faktors Arbeit. Die Nutzung lokaler Ressourcen (von der Biomasse bis zur Abwärme) schafft lokale Arbeitsplätze. Die starke Dienstleistungsverflechtung wissensintensiver Produktionsweisen schafft dem Überangebot an Hochschulabsolventen Beschäftigungsmöglichkeiten.

Ökologische Strukturpolitik ist auf Branchenebene vor allem eine Modernisierungsstrategie

Fortsetzung S. 2
VÖW-Jahrestagung 1989

Staatliche

Wirtschaftspolitik als Umweltzerstörung?

Der Staat in der

Umweltverträglichkeitsprüfung

7.-9. Juni 89, Haus der Kirche, Goethestr. 27-30, 1000 Berlin 12

Die Tagung soll „den" Staat (schwerpunktmäßig den Zentralstaat) als aktives Gestaltungselement der Umwelt in den Mittelpunkt rücken-und zwar in zweifacher Weise:

Erstens soll verdeutlicht werden, daß der Staat die Umwelt durch explizit umweltpolitische Maßnahmen beeinflußt. Allerdings wird die Umwelt entscheidender geprägt durch das, was er im Bereich der Finanzpolitik, der Verkehrs-, Energie- und Strukturpolitik, also den Kernbereichen seiner Wirtschaftspolitik, tut (oder läßt). Hier tritt der Staat vor allem als Mitverursacher von Schäden auf in einer Weise, die seine Rolle als umweltpolitische Reparaturinstanz verblassen läßt.

Zweitens ist damit der Staat aber auch als möglicher Akteur für eine ökologieverträgliche Wirtschaftsweise thematisiert. Als solcher ist er der legitime Adressat für ökologieverträgliche Alternativvorschläge in den einzelnen Bereichen seiner Wirtschaftspolitik. Dies soll zum Anlaß genommen werden, die Perspektiven für eine ökologisch-ökonomische Alternativberichterstattung auszuloten. Im Blick auf das übergreifend angelegte Vorhaben der ökologisch-ökonomischen Stellungnahmen soll die Tagung daher auch ein Beitrag zur diesbezüglichen Selbstverständigung des IÖW/VÖWUmfeldes sein.

Das Eingangsreferat „Der Staat als umweltpolitischer Akteur: Problemlöser und Problemproduzent" wird Martin Jänicke halten. Dieser organisiert auch gemeinsam mit Frank Beckenbach die Tagung.
Die IÖW-Jahrestagung 1989 wird aller Voraussicht nach vom 25. bis 26. November in Düsseldorf stattfinden. Thema: ,Regionale Entwicklungskonzepte ${ }^{66}$. 


\section{Fortsetzung von S. 1}

(intrasektorale Strukturpolitik). Hier bieten sich den Einzelgewerkschaften besondere Handlungsmöglichkeiten. Sie zielt aber auch auf eine Verschiebung zwischen den Branchen ab (intersektorale Strukturpolitik), eine Aufgabe, die im gesamtgewerkschaftlichen wie gesamtgesellschaftlichen Konsens anzugehen ist.

Die Vorstudie konzentriert sich auf die derzeitigen Ausgangsbedingungen in ausgewählten $\mathrm{Be}$ reichen von hoher ökologischer Bedeutung.

In der Infrastruktur hat sich bis 1987 ein umweltentlastender intersektoraler Wandel kaum vollzogen. Nur im Bereich Steine/Erden ergab sich ein gewisser. Wandlungsproze $B$ in dieser Richtung (wie auch anderswo). Innerhalb der Branchen fällt der überaus rasche Stromverbrauchszuwachs pro Beschäftigten auf, der dafür spricht, daß Rationalisierungsstrategien sich besonders stark auf den Faktor Arbeit und kaum auf den Energieverbrauch konzentrieren. Der Primärenergieverbrauch insgesamt steigt sogar stärker an, als die Energie-Enquetekommission des Abgeordnetenhauses 1983 unter der Annahme errechnete, daß keine besonderen Energiesparmaßnahmen ergriffen werden!

Der Dienstleistungssektor ist zwar gewachsen. Aber er ist nur unzureichend in die vorhandene Wirtschaftsstruktur integriert und ist kaum ein Faktor ihrer Modernisierung. Es dominiert ein Typus von ,alten“ Dienstleistungen (z.B. im öffentlichen Sektor).

In der Flächennutzung kommen strukturelle Defizite der Stadt im Vergleich zu anderen entwikkelten Ballungsräumen besonders deutlich zum Ausdruck. Dies gilt für die anomale Größe der Verkehrsfläche ebenso wie für den industriellen Flächenbedarf. Während in hochentwickelten Industrieregionen (z.B. Tokio) die Industriefläche trotz starkem Wirtschaftswachstum zurückging, gilt für den (bisherigen) Senat ein langfristiger Flächenbedarf von zusätzlichen 300 ha. Hier sind klare Alternativen möglich. Stadtgerechte Industrien (urban-type industries) benötigen z.B. keine besonderen Industrieflächen und sind auch sparsamer im Flächenverbrauch.

In der weiteren Forschung wird ausführlich auf die Instrumente einer alternativen Strukturpolitik für die Stadt einzugehen sein. Die Berlinförderung hat besonders nachteilige Industriestrukturen entstehen lassen. Dieses Förderinstrument könnte aber auch im alternativen Sinne genutzt werden und so Berlin vor anderen Städten auszeichnen. Das Gewicht der ökonomischen Instrumente müßte sich aber teilweise von den Ausgaben hin zu den Einnahmen des Staates bzw. seiner Versorgungsbetriebe verlagern. Der Steuerung über ökologische Steuern, Abgaben und Tarife wird hierbei eine den Haushalt wie die Umwelt entlastende Rolle zukommen.

Ausführlich untersucht werden soll auch die Rolle anderer Instrumente der Umwelt- und der Wirtschaftspolitik. Dabei wird u.a. die Bedeutung partizipativer Instrumente herausgestellt werden, die von der Verbandsklage bis zu ökologischen Mitbestimmung der Gewerkschaften reichen.

\section{Institut für ökologische Wirtschaftsforschung GmbH Giesebrechtstraße 13 1000 Berlin 12 Tel.: $030 / 8825281$}

\section{PUBLIKATIONEN DES IÖW}

Reinhard Pfriem (Hg.): Ökologische Unternehmenspolitik. 280 S., DM 25,-, (verbilligt/portofrei) Abschätzung der Folgen eines Ausstiegs aus der Kernenergie. Gutachten im Auftrag des Bundeswirtschaftsministeriums, 260 S., DM 45,-

\section{Schriftenreihe des Iöw}

1/86: Auswege aus dem industriellen Wachstumsdilemma. Broschüre zur IÖW-Tagung Nov. 1985, 144 S., DM 15 .

2/87: Umweltentlastung durch wirtschaftlichen Strukturwandel? Dokumentation der IÖW-Tagung Nov. 1986, 150 S., DM 28,

3/87: Alternative Energiepolitik in der DDR und in West-Berlin. Möglichkeiten einer exemplarischen Kooperation in Mitteleuropa. Gutachten, 120 S., DM 20,-

4/87: Die Auswirkungen der geplanten Müllverbrennungsanlage sowie die Entwicklung und Bewertung eines alternativen Abfallkonzepts für Berlin-West. Gutachten, 141 S., DM 18,

5/87: Nicholas Georgescu-Roegen: The Entropy Law and the Economic Process in Retrospect. IÖW-eigene Übersetzung ins Deutsche mit Geleitwort, 70 S., DM 20,-

6/87: Bodenschutzpolitik und ökologische Wirtschaftsforschung. Dokumentation der VÖW-Tagung Juni 1987, 105 S., DM 20,-

7/87: B.P. Priddat, J. Meran, St. Zundel: Ökologische Ethik der Ökonomíe? 65 S., DM 15,-

8/87: Jan C. Bongaerts, R. Andreas Kraemer: Haftung und Versicherung von Umweltschäden. IÖW-eigene Übers., 50 S., DM 15,-

9/87: Eberhard K. Seifert: Wirtschaftsethik in ökologischer Absicht, 50 S., DM 15,

10/88: Die regionale Wirtschaftsförderung - Bestandsaufnahme und Alternativen. Eine Untersuchung am Beispiel Niedersachsens. Gutachten, erstellt von Frank Beckenbach. 120 S., DM 28,

11/88: Birger P. Priddat: Ökonomie und/oder Natur. Zur Abschätzung ökonomischer Reichweiten ökologischer Ideen, 60 S., DM 20,-

12/88: Jürgen Freimann, Reinhard Pfriem (Hg.): Ökologische Betriebswirtschaftslehre und -praxis? 70 S., DM 28,-

13/88: Reinhard Pfriem: Ökologische Unternehmensführung, ca. 70 S., ca. DM 22,-

14/88: Jürgen Koch, Berthold Pechan: Ökologischer Stadtumbau Berlin. Gutachten, 70 S., DM 22,-

15/88: Ludwig Trepl (Hg.): Beiträge zum ökologischen Diskurs, 100 S., DM 28,-

16/88: Ralf Antes: Umweltschutzinnovationen als Chancen des aktiven Umweltschutzes für Unternehmen im sozialen Wandel, 159-S., DM 35,-

17/88: Jürgen Koch, Jürgen Pöschk: Produktion, Produkte und Forschungsprojekte im Bereich Umwelttechnologien der Berliner Metallindustrie, in Instituten und Forschungseinrichtungen, 58 S., DM 20,-

18/88: Burkhart Heinz (Hg.): Ökomarketing, 149 S., DM 35 ,

19/88: Juan Martinez-Alier: Towards a History of Ecological Economics, Deutsche Übersetzung mit Kommentar von Frank Beckenbach, ca.60 S., ca. DM 22,

20/88: Frank Beckenbach, Ulrich Hampicke, Werner Schulz: Möglichkeiten und Grenzen der Monetarisierung von Natur und Umwelt, ca. 60 S., ca. DM 20,-

21/88: Harald Müller-Witt, Frank Springmann: Ökologischer Umbau des Steuersystems, 79 S., DM 23,-

22/88: Hansjörg Herr, Klaus Voy: Neuorientierung der bundesdeutschen Währungs- und Finanzpolitik im internationalen Rahmen, 205 S., DM 38,

23/89: Eberhard Schmidt (Hg.): Ökologische Produktionskonzepte, ca. 120 S., ca. DM 20,-

24/89: Christoph Nitschke, Umweltrelevante Qualifikationen unter besonderer Berücksichtigung von Betrieben der Berliner Metallindustrie, 64 S., DM 20,-

Mitglieder der Vereinigung für ökologische zuzüglich Versandkosten

Wirtschaftsforschung (VÖW) erhalten 20 Prozent Rabatt !

$4 \times$ jährlich erscheint der

INFORMATIONSDIENST für ökologische Wirtschaftsforschung.

Normalabo: DM 20,- pro Jahr $\star$ Probeexemplar kostenlos erhältlich

\section{Vereinigung für ökologische Wirtschaftsforschung (VöW) e.V.}

Konto: 790010070 Sparkasse Berlin, BLZ 100500 00. Vorstand: Dr. Michaele Schreyer, Dipl.-Volksw. Sabine Spelthahn, Prof. Dr. Martin Jänicke, Prof. Dr. Rainer Knigge, Prof. Dr. Eberhard Schmidt

Institut für ökologische Wirtschaftsforschung (IÖW) $\mathrm{GmbH}$

Konto: 790010135 Sparkasse Berlin, BLZ 10050000 Beirat: Dr. Irene Schöne, Dr. Jan C. Bongaerts, Dipl. Volksw. Burghard Flieger, Dr. Arnim von Gleich, Dr. Eckart Hildebrandt, Prof. Dr. Martin Jänicke, Dr. Christian Leipert, Dr. Lutz Mez, Dr. Joachim Müller, Dr. Reinhard Pfriem, Dr. Eberhard K. Seifert, Prof. Dr. Udo E. Simonis, Prof. Dr. Burkhard Strümpel, Dr. Otto Ullrich, Dr. Helmut Wiesenthal 
(c) 20Io Authors; licensee IÖW and oekom verlag. This is an article distributed under the terms of the Creative Commons Attribution Non-Commercial No Derivates License (http://creativecommons.org/licenses/by-nc-nd/3.o/), which permits unrestricted use, distribution, and reproduction in any medium, provided the original work is properly cited. 